\title{
Optical fiber dosimetry at the Tesla Test Facility (TTF)
}

H. Henschel, O. Köhn, M. Körfer, T. Stegmann, K. Wittenburg, and F. Wulf

Citation: AIP Conference Proceedings 546, 647 (2000);

View online: https://doi.org/10.1063/1.1342641

View Table of Contents: http://aip.scitation.org/toc/apc/546/1

Published by the American Institute of Physics 


\title{
Optical Fiber Dosimetry at the Tesla Test Facility (TTF)
}

\author{
H. Henschel ${ }^{1}$, O.Köhn ${ }^{1}$, M. Körfer ${ }^{3}$, T. Stegmann ${ }^{3}$, \\ K. Wittenburg ${ }^{3}$, F. Wulf ${ }^{2}$ \\ ${ }^{\prime}$ Fraunhofer Gesellschaft INT, Germany \\ ${ }^{2}$ Hahn Meitner Institut HMI, Germany \\ ${ }^{3}$ Deutsches Elektronen Synchrotron DESY, Germany
}

\begin{abstract}
Ionizing radiation leads to a degradation of the light transmitting properties of fiber optic cables. These effects usually place restrictions on where they can be used in a particle accelerator facility. These effects can also be used to our advantage, the losses from a particle beam create wavelength dependant increases of attenuation by absorption and scattering which can be measured using Optical Time Domain Reflectometery (OTDR). From these measurements a measure of the radiation dose received can be inferred.
\end{abstract}

Optical fibers are used in a wide field of applications, where their degradation by ionizing radiation is often an unwanted effect. The main radiation effect is a wavelength-dependent increase of attenuation by absorption and scattering processes. Despite the fact that the different types of modern telecommunication fibers show strongly increased radiation hardness, compared with the early ones, there still exist some single-mode (SM) and multi-mode graded index (MM GI) fibers with high and only slowly annealing loss increase. Such fibers can be used for in situ local radiation dosimetry also at particle accelerators, to observe the emission of radiation along the beam line where radiation-sensitive equipment might have to be installed.

The advantages of optical fiber dosimetry are that:

- Modern fibers can be produced with highly identical composition and quality in great lengths, enabling the radiation control of very lengthy objects or spacious areas.

- The dosimeter sensitivity can be adjusted to the dose or dose rate of interest by selection of wavelength or fiber type. The radiation-induced attenuation increases from the minimum around $1100 \mathrm{~nm}$ towards about $670 \mathrm{~nm}$ (red) or $450 \mathrm{~nm}$ (blue) by orders of magnitude. Ge-doped MM GI fibers co-doped with Phosphorus (P) show medium radiation sensitivity, whereas the Rare Earth doped SM fibers that are used for fiber amplifiers or lasers show tremendous increase of loss.

- The dosimeter dimensions can be very small. Bare (i.e. uncabled) fibers usually have a diameter of only $0.25 \mathrm{~mm}$, so that they can, for example, measure the dose in otherwise inaccessible, narrow slits.

CP546, Beam Instrumentation Workshop 2000, edited by Kenneth D. Jacobs and R. Coles Sibley III (C) 2000 American Institute of Physics 1-56396-975-0/00/\$17.00 
One task of fiber optic dose sensors at TTF could be the detection of radiation dose levels along accelerator sections, caused by the dark current due to field emission in one of the super-conducting accelerator cavities and the RF laser gun, or by beam losses at certain positions. Such a dosimeter could be verified by laying the selected fiber (e.g. (Ge+P)-doped MM GI) along the accelerator section of interest. The attenuation along the fiber can be measured with a commercial Optical Time Domain Reflectometer (OTDR) operating at $850 \mathrm{~nm}$. Sections with higher radiation exposure attenuate the light emitted into and coming back from the fiber section behind, leading to a step in the OTDR trace. The height of the step is proportional to the radiation dose.

Another task would be a continuous dose measurement at one or several or even a bigger fraction of the radiation-sensitive undulator magnets. This can be verified by laying the same fiber type meander-like through the magnet system and measuring the attenuation with an OTDR of high local resolution. Another solution would be to place several windings of separate fibers around all magnets of interest and measure the transmission loss in each individual branch with a multisensor optical power meter. Furthermore one could use the luminescence light (predominantly Cerenkov light) that will be generated in optical fibers during unallowed high radiation emission from a certain accelerator section to initiate a rapid switch off, in order to prevent serious damage during longer disadjustment periods.

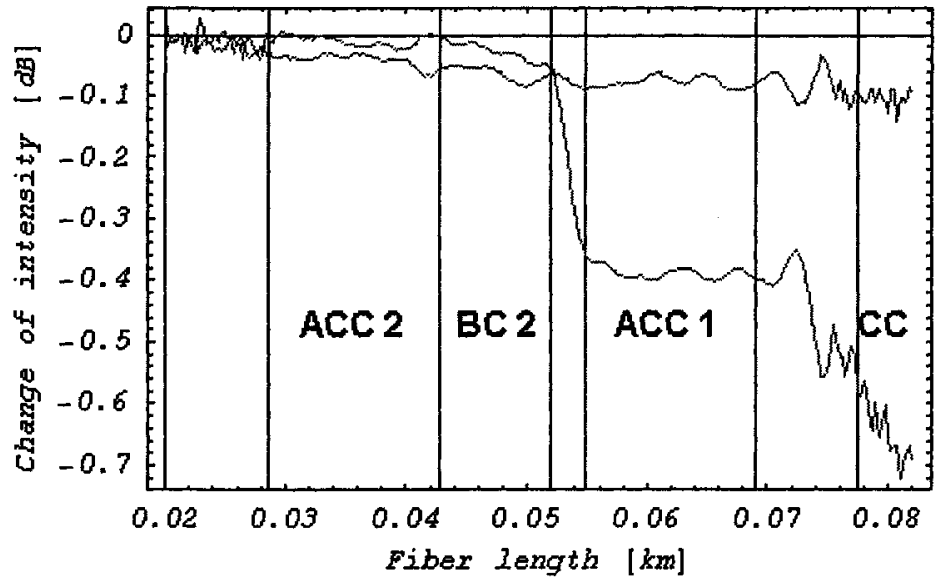

Figure 1: Response of a dosimeter fiber during two different accelerator operation conditions. The upper curve shows the degradation within two weeks with normal operation parameters (difference of the OTDR traces after and before irradiation). The lower curve shows the same difference after a week with extensive dark current losses in section $\mathrm{CC}$ and in front of $\mathrm{BC} 2$. The degradation is concentrated at locations where the dark current is expected to be lost. The position resolution is about one meter. It is a convolution of the loss distribution and the position resolution of the readout system. The accumulated dose in the affected areas was on the order of $10 \mathrm{~Gy}$. 
Preliminary dose measurements along an accelerator section of TTF as well as at the undulator magnets were performed at the beginning of the year 2000, with both measurement systems (OTDR and optical power meter). The preliminary measurements performed with less suited OTDRs and not optimized optical fibers showed significant attenuation steps (Fig. 1). The optical power meter system promised to detect even lowest radiation levels at the magnets. Detailed results of these preliminary tests will be documented soon in [1]. An improved and more extensive pilot project at TTF that began in May 2000 will be documented elsewhere.

One disadvantage of optical fiber dosimeters is that the increase of attenuation with dose saturates after dose values of several kGy (dependent on fiber type). Since such dose values could be accumulated within relatively short periods, at least at certain critical locations, the fibers would have to be exchanged for new ones relatively often. However, it is known that the radiation-induced fiber loss can be annealed by high temperatures and/or higher light intensities (Photobleaching). During preliminary investigations with $(\mathrm{Ge}+\mathrm{P})$-doped MMGI fibers the loss increase could be reduced to about $25 \%$ of its initial value. The following irradiation caused exactly the same increase of loss with dose as the first one; i.e. the calibration curve remained the same.

\section{REFERENCES}

1. TESLA Report in preparation: "Preliminary Trials with Optical Fiber Dosimeters at TTF". 